\title{
VERZEICHNIS DER SCHRIFTEN VON DIETER JANIK ZUR SPANISCHAMERIKANISCHEN LITERATUR
}

\author{
Monographien
}

(1976): Magische Wirklichkeitsauffassung im hispanoamerikanischen Roman des 20. Jahrhunderts. Geschichtliches Erbe und kulturelle Tendenz. Con un resumen en español, Tübingen: Niemeyer.

(1989) zusammen mit Wolf Lustig: Die spanische Eroberung Amerikas: Akteure, Autoren, Texte. Eine kommentierte Anthologie von Originalzeugnissen, Frankfurt am Main: Vervuert.

(1992): Stationen der spanischamerikanischen Literatur- und Kulturgeschichte. Der Blick der anderen - der Weg zu sich selbst, Frankfurt am Main: Vervuert.

(1995): Die Anfänge einer nationalen literarischen Kultur in Argentinien und Chile. Eine kontrastive Studie auf der Grundlage der frühen Periodika (1800-1830), Tübingen: Narr.

\section{Herausgeberschaften}

(1994) (Hrsg.): Die langen Folgen der kurzen Conquista. Auswirkungen der spanischen Kolonisierung Amerikas bis heute, Frankfurt am Main: Vervuert.

(1998) (Hrsg.): La literatura en la formación de los Estados hispanoamericanos (18001860), Madrid / Frankfurt am Main: Iberoamericana / Vervuert.

\section{Aufsätze}

(1970): »Ercilla, lector de Lucano«, in: Instituto Central de Lenguas (Hrsg.): Homenaje a Ercilla (1969), Universidad de Concepción, pp. 83-109.

(1972): »Der >realismo mágico< - zur Bedeutung des Magischen im hispanoamerikanischen Gegenwartsroman«, in: Hösle, Johannes / Eitel, Wolfgang (Hrsg.): Beiträge zur vergleichenden Literaturgeschichte. Festschrift für Kurt Wais zum 65. Geburtstag, Tübingen, pp. 375-387.

(1975a): »La estructura comunicativa de la obra narrativa - un modelo semiológico«, Acta Scientifica (Universidad Católica de Córdoba) 5, pp. 15-38.

(1975b): »Introducción a la lingüística del texto en su rama alemana. Su importancia para una Ciencia de la Literatura «, Acta Scientifica 5, pp. 61-75.

(1975c): »Las investigaciones sobre la literatura hispanoamericana en la República Federal Alemana en la última década«, Acta Scientifica 5, pp. 103-113.

(1975d): »La concepción mágica de la realidad en la narrativa hispanoamericana del siglo XX«, Acta Scientifica 5, pp. 115-129.

(1975e): „El análisis temático de la obra narrativa y el problema de la estructura del contexto cultural«, Acta Scientifica 5, pp. 149-161.

(1978): „Gabriel García Márquez«, in: Eitel, Wolfgang (Hrsg.): Lateinamerikanische 
Literatur der Gegenwart in Einzeldarstellungen, Stuttgart: Kröner, pp. 330-360.

(1980a): »Las investigaciones sobre literatura latinoamericana en la República Federal

Alemana en la última década«, Iberoromania 5, pp. 215-224.

(1980b): »Tesis de habilitación y tesis de doctorado realizadas en las universidades de la República Federal de Alemania, de la República Democrática Alemana y de Austria sobre temas de lengua y literatura hispanoamericanas y brasileñas (19451979)«, Iberoromania 5, pp. 225-235.

(1980c): »Zwei Wiegenlieder von Gabriela Mistral«, in: Oroz Arizcuren, Francisco J. (Hrsg.): Romania cantat. Gerhard Rohlfs zum 85. Geburtstag gewidmet: Lieder in alten und neuen Chorsätzen mit sprachlichen, literarischen und musikwissenschaftlichen Interpretationen, 2 Bde., Tübingen, Bd. 2, pp. 273-275.

(1980d): »Kulturzusammenstoß und Kulturschock in der Neuen Welt«, Hispanorama 24 , pp. 30-33.

(1980e): »[Beitrag] «, in: Benecke, Dieter / Domitra, Michael / Mols, Manfred (Hrsg.): Integration in Lateinamerika. Beiträge des Symposiums der Arbeitsgemeinschaft Deutsche Lateinamerikaforschung, München: Fink, pp. 353-357.

(1982a): m[...] verdienet nicht ein Mensch zu sein.«Fremdverständnis und Selbstverständnis der Menschen und Kulturen zum Zeitpunkt der Conquista«, in: Arbeitskreis Dritte Welt der Johannes Gutenberg-Universität Mainz (Hrsg.): Menschenrechte und Menschenbild in der Dritten Welt. Ringvorlesung Wintersemester 1978/79, Frankfurt am Main: Vervuert, pp. 61-80.

(1982b): „Vicente Huidobro und César Vallejo: Zwei Außenseiter der europäischen Avantgarde aus Spanischamerika«, in: Warning, Rainer / Wehle, Winfried (Hrsg.): Lyrik und Malerei der Avantgarde, München: Fink, pp. 193-209.

(1982c): „Signos - Relaciones semióticas - Decodificación de Signos. Semiótica literaria para el lector «, Acta Literaria 7, pp. 5-16.

(1983): »Drei Zentralmotive der dichterischen Einbildungskraft des Erzählers Gabriel García Márquez: La casa - El huracán - La muerte«, in: López de Abiada, José Manuel / Heydenreich, Titus (Hrsg.): Iberoamérica. Historia, sociedad, literatura. Homenaje a Gustav Siebenmann, 2 Bde., München: Fink, Bd. 1, pp. 369-387.

(1984a): ")Civilización y Barbarie. Die Entwicklungsproblematik in der kulturkritischen Literatur Spanischamerikas im 19. und 20. Jahrhundert«, in: Oroz Arizcuren, Francisco J. (Hrsg.): Navicula Tubingensis. Studia in honorem Antonii Tovar, Tübingen, Narr, pp. 221-232.

(1984b): »Drei Zentralmotive der dichterischen Einbildungskraft des Erzählers Gabriel García Márquez: La casa - El huracán - La muerte«, in: Stefanovics, Tomás (Hrsg.): Symposion über Gabriel Garcia Márquez, Nürnberg, pp. 34-46.

(1986a): »[Beitrag]«, in: Lateinamerika - Herausforderungen an Politik und Wissenschaft. Kolloquium der Arbeitsgemeinschaft Deutsche Lateinamerikaforschung (ADLAF), 8. Januar 1986. Anuario 1, Münsteraner Schriften zur LateinamerikaForschung, pp. 25-29.

(1986b): „Imaginación mítica en Cien años de soledad de Gabriel García Márquez, Daimón de Abel Posse y Casa de Campo de José Donoso«, in: Les Mythologies hispaniques dans la seconde moitié du XXe siècle. Actes du colloque international du Centre d'études et de recherches hispaniques du XX siècle, Dijon, pp. 77-84.

(1986c): »Momotombo ou la dimension mythique de Victor Hugo dans la poésie hispa-

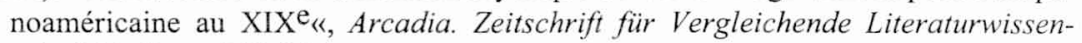
schaft 21: 3, pp. 233-244. 
(1987a): »El Periquillo Sarniento de J. J. Fernández de Lizardi: Una normativa vacilante (Sociedad - Naturaleza y Religión - Razón)«, Ibero-Amerikanisches Archiv 13: 1, pp. 49-60.

(1987b): »Momotombo ou la dimension mythique de Victor Hugo dans la poésie hispa-

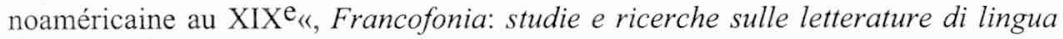
francese 13.

(1988): »Ercilla, lector de Lucano«, in: Becker, Félix (Hrsg.): América Latina en las letras y ciencias sociales alemanas, Caracas: Monte Ávila, pp. 371-399.

(1989a): »)Epitafio y Epitafios en la obra de Gonzalo Rojas«, Ibero-Amerikanisches Archiv 15: 1, pp. 57-64.

(1989b): »Mythische Imagination in Cien años de soledad von Gabriel García Márquez, Daimón von Abel Posse und Casa de campo von José Donoso«, in: Wentzlaff-Eggebert, Christian (Hrsg.): Realität und Mythos in der lateinamerikanischen Literatur. Akten des Internationalen Literatursymposions in Lindau (22.-24.3.1984), Köln / Wien: Böhlau, pp. 279-289.

(1989c): »Das Kains-Motiv in spanischamerikanischen Romanen des 20. Jahrhunderts«, in: Link, Franz (Hrsg.): Paradeigmata. Literarische Typologie des Alten Testaments, 2 Bde., Berlin: Duncker \& Humblot, Bd. 2: 20. Jahrhundert, pp. 577-589.

(1990): »La valoración múltiple del indio en La Araucana de Alonso de Ercilla«, in: Consejo Superior de Investigaciones Científicas (Hrsg.): La imagen del indio en la Europa moderna, Sevilla.

(1991): »El perfil >romántico< de la literatura hispanoamericana en la época de su florecimiento (1945-1975)«, in: Strosetzki, Christoph / Botrel, Jean-François / Tietz, Manfred (Hrsg.): Actas del I Encuentro Franco-Alemán de Hispanistas (Mainz 9.12.3.1989), Madrid / Frankfurt am Main: Iberoamericana / Vervuert, pp. 180-186.

(1990): " La noción de sociedad en el pensamiento de Lizardi y de sus contemporáneos«, Cahiers des Amériques Latines 10, pp. 39-48.

(1992): „Gabriel García Márquez: Cien años de soledad«, in: Roloff, Volker / Wentzlaff-Eggebert, Harald (Hrsg.): Der hispanoamerikanische Roman, 2 Bde., Darmstadt: Wissenschaftliche Buchgesellschaft, Bd. 2, pp. 132-145.

(1994a): "La experiencia de la Violencia: problemas de su transposición estética«, in: Kohut, Karl (Hrsg.): Literatura colombiana hoy. Imaginación y barbarie, Madrid / Frankfurt am Main: Iberoamericana / Vervuert, pp. 139-146.

(1994b): »J. B. Alberdi: Peregrinación de Luz del Día o viaje y aventuras de la Verdad en el Nuevo Mundo: análisis y parodia de la realidad hispanoamericana desde la Independencia«, in: Schönberger, Axel / Zimmermann, Klaus (Hrsg.): De orbis Hispani linguis litteris historia moribus. Festschrift für Dietrich Briesemeister zum 60. Geburtstag, 2 Bde., Frankfurt am Main: Domus Editoria Europaea, Bd. 2, pp. 14671473.

(1994c): »Die neuen Menschen der Neuen Welt. Zur gesellschaftlichen und kulturellen Rolle der mestizos«, in: Janik, Dieter (Hrsg.): Die langen Folgen der kurzen Conquista. Auswirkungen der spanischen Kolonisierung Amerikas bis heute, Frankfurt am Main: Vervuert, pp. 49-73.

(1995a): »Der Staat im Spiegel der spanischamerikanischen Literatur«, in: Mols, Manfred / Thesing, Josef (Hrsg.): Der Staat in Lateinamerika, Mainz: von Hase \& Koehler, pp. 303-316.

(1995b): »El Estado en el espejo de la literatura hispanoamericana«, in: Mols, Manfred / Thesing, Josef (Hrsg.): El Estado en América Latina, St. Augustin bei Bonn / Bue- 
nos Aires: Konrad-Adenauer-Stiftung, A.C. - CIEDLA, pp. 343-357.

(1995c): »Literatur als gesellschaftliche Notwendigkeit der unabhängigen Staaten Spanischamerikas «, in: Neubert, Dieter / Thimm, Andreas (Hrsg.): Kunst, Literatur und Gesellschaft. Zur Rezeption und Bedeutung von Kunst und Literatur in der Dritten Welt, Mainz, pp. 31-44.

(1995d): »El concepto de periodización en la renovada historiografía literaria hispanoamericana«, Nuevo texto crítico 14/15, pp. 107-113.

(1997a): »Sor Juana Inés de la Cruz: `Este, que ves, engaño colorido«, in: Tietz, Manfred (Hrsg.): Die spanische Lyrik von den Anfängen bis 1870, Frankfurt am Main: Vervuert, pp. 503-511.

(1997b): »Sor Juana Inés de la Cruz«, in: Hölz, Karl / Jüttner, Siegfried / Stillers, Rainer / Strosetzki, Christoph (Hrsg.): Sinn und Sinnverständnis. Festschrift für Ludwig Schrader zum 65. Geburtstag, Berlin: Schmidt, pp. 225-232.

(1998): "Desde la literatura hasta las bellas letras. Los principios de una literatura nacional en Nueva Granada (Colombia) al final de la época colonial y en el primer período de la Independencia, reflejados en los periódicos (1791-1859)«, in: Janik, Dieter (Hrsg.): La literatura en la formación de los Estados hispanoamericanos (1800-1860), Madrid / Frankfurt am Main: Iberoamericana / Vervuert, pp. 197-217.

(2000a): »Observaciones sobre el status de la literatura colonial: el siglo XVI«, in: Kohut, Karl / Rose, Sonia V. (Hrsg.): La formación de la cultura virreinal, Madrid / Frankfurt am Main: Iberoamericana/Vervuert, pp. 125-131.

(2000b): »Periodismo y literatura: su alianza en la época de la Independencia bajo el signo de la Ilustración (Argentina, Chile, Colombia)«, Acta Literaria 25, pp. 37-47.

(2000c): »El Diálogo de los Porteros y otros diálogos políticos de la Revolución de la Independencia de Chile«, in: Gunia, Inke / Niemeyer, Katharina / Schlickers, Sabine / Paschen, Hans (Hrsg.): La modernidad revis(it)ada. Literatura y cultura latinoamericanas de los siglos $X I X$ y $X X$. Estudios en homenaje a Klaus MeyerMinnemann, Berlin: Tranvía, pp. 41-54.

(2001): »Respuesta y pregunta: la necesidad de entender en el encuentro de españoles y aztecas, partiendo de Coloquios y Doctrina Cristiana de Fray Bernardino de Sahagún (1524-1564)«, in: Castañeda, Felipe / Vollet, Matthias (Hrsg.): Concepciones de la Conquista. Aproximaciones interdisciplinarias, Santafé de Bogotá: Uniandes, pp. 159-176.

\section{Rezensionen}

(1968): »[Referat in spanischer Sprache über 2 Vorträge von Eugenio Coseriu: 1. Lenguaje y Poesia, 2. Origen y sentido de la lingüistica actual]«, Atenea CXIX, pp. 495-497.

(1971): »Leo Pollmann, >Der Neue Roman in Frankreich und Lateinamerika<, Stuttgart 1968«, Zeitschrift für französische Sprache und Literatur LXXI: 1, pp. 86-89.

(1972): »Bärbel Brinckmann, >Quellenkritische Untersuchungen zum mexikanischen Missionsschauspiel (1533-1732)<, Diss. Hamburg 1970«, Kritikon Litterarum 1, pp. 29-30.

(1981a): »Pedro Ramírez Molas, `Tiempo y narración. Enfoques de la temporalidad en Borges, Carpentier, Cortázar y García Márquez‘, Madrid 1978«, Iberoromania 13, pp. 114-115.

(1981b): „Edelweis Serra, `Tipología del cuento literario. Textos hispanoamericanos‘, 
Madrid 1978«, Iberoromania 13, pp. 115-116.

(1981c): »Karsten Garscha / Horst G. Klein, >Einführung in die Lateinamerikastudien am Beispiel Peru<, Tübingen 1979«, Romanistisches Jahrbuch 32, pp. 404-405.

(1984a): »Víctor Farías, ,Los Manuscritos de Melquíades. Cien años de soledad, burguesía latinoamericana y dialéctica de la reproducción ampliada de negación‘, Frankfurt am Main 1981«, Iberoromania 19, pp. 123-125.

(1984b): »Michael Palencia-Roth, ,Gabriel García Márquez. La línea, el círculo y las metamorfosis del mito<, Madrid 1983«, Romanistisches Jahrbuch 35, pp. 339-341.

(1984c): »)Studii Latinoamericani 81: la narrativa latinoamericana contemporánear, a cura di Giulia Lanciani e Giuseppe Bellini, L’Aquila 1982«, Romanische Forschungen $96: 3$, pp. 379-381.

(1986a): »John S. Brushwood, >La novela hispanoamericana del siglo XX. Una vista panorámica<, traducción de Raymond L. Williams, Mexiko 1984«, Romanische Forschungen 98: 3/4, pp. 487-489.

(1986b): »Frank Pierce, >Alonso de Ercilla y Zúñiga〈, Amsterdam 1984«, GermanischRomanische Monatsschrift 36: 3, pp. 363-365.

(1987): „Leo Pollmann, ,Geschichte des lateinamerikanischen Romans. I: Die literarische Selbstentdeckung (1810-1929)‘, Berlin 1982«, Germanisch-Romanische Monatsschrift 37: 3, pp. 348-350.

(1988): »)Latin American Literary Review. Special Issue, vol. XV, January-June 1987, no. 29. The Boom in Retrospect: A Reconsideration<, ed. by Yvette E. Miller and Raymond Leslie Williams, Pittsburgh«, Romanische Forschungen 100: 4, pp. 486488.

(1991a): »J. Walker, >Rivera: La vorágine`, London 1988«, Romanische Forschungen 103: 2/3, pp. 356-357.

(1991b): »Hispanic Issues. Vol. 4: 1492-1992: Re/Discovering Colonial Writing८, ed. by René Jara and Nicholas Spadaccini, Minneapolis 1989«, Romanische Forschungen 103: 4, pp. 515-517.

(1991c): »Karl Hölz (Hrsg.), >Literarische Vermittlungen: Geschichte und Identität in der mexikanischen Literatur, Akten des Kolloquiums Trier«, Tübingen 1988«, Zeitschrift für Romanische Philologie 107: 5/6, pp. 719-721.

(1992): »Montserrat Ordóñez (Hrsg.), >La vorágine: textos críticos«, Bogotá 1987«, Iberoromania 36 , pp. 121-123.

(1993): »David William Foster, >Alternate Voices in the Contemporary Latin American Narrative $\prec$, Columbia 1985«, Iberoromania 37, pp. 150-152.

(1995a): »Sonia Mattalia, `La figura en el tapiz. Teoría y práctica narrativa en Juan Carlos Onetti<, London 1990«, Zeitschrift für Romanische Philologie 111: 2, pp. 314316.

(1995b): »Djelal Kadir, >The Other Writing. Postcolonial Essays in Latin America's Writing Culture،, West Lafayette (Indiana) 1993«, Romanische Forschungen 107: 1/2, pp. 271-272.

(1996): »Luis Muñoz González / Dieter Oelker Link, >Diccionario de movimientos y grupos literarios chilenos`, Concepción 1993«, Notas 1: 3, pp. 126-128.

(2000): „Erik Camayd-Freixas, ,Realismo mágico y primitivismo. Relecturas de Carpentier, Asturias, Rulfo y García Márquez`, Lanham / New York / Oxford 1998«, Notas 7: 2, pp. 118-119. 


\section{Dieter Janik}

\section{Varia}

(1976): »Weltliteratur aus Lateinamerika: Mit ZDF und Kanu über den Orinoco«, Börsenblatt für den Deutschen Buchhandel, Themensondernummer Lateinamerika 32. Jahrgang, 2. September 1976, Frankfurter Ausgabe, pp. 18-23.

(1986): „Geistige Ausstrahlung von Willigis ins Land der Azteken«, Mainzer Allgemeine Zeitung, 7./8. Juni, p. 7. 